\title{
Is secondary tracheomalacia associated with persistent lower respiratory tract inflammation and subclinical infection?
}

\author{
Antonino Capizzi ${ }^{1}$, Michela Silvestri ${ }^{1}$, Giovanni Rossi ${ }^{2}$, and Oliviero Sacco ${ }^{1}$ \\ ${ }^{1}$ Istituto Giannina Gaslini \\ ${ }^{2} \mathrm{G}$. Gaslini Institute
}

December 13, 2020

\begin{abstract}
Background. In secondary tracheomalacia due to mediastinal vascular anomalies one of the most prevalent symptom is recurrent lower respiratory tract (LRT) infections, related to defective airway clearance. Whether this condition could result in persistent LRT inflammation and subclinical infection is not known. Patients and methods. Children with tracheomalacia due to mediastinal vascular anomalies, recurrent $(>3 / y)$ LRT infections were evaluated while in stable condition. Computed tomography (CT) scan and bronchoscopy with bronchoalveolar lavage (BAL) were performed. Results. 31 children were included in the study: 21 with aberrant innominate artery (AIA), 4 with right aortic arch (RAA) and 1 with double aortic arch (DAA) and 5 with AIA associated with RAA. Cytological evaluation of BAL fluid showed increased neutrophil percentages and normal lymphocyte and eosinophil proportions. Microorganism growth was detected in $35.5 \%$ of BAL samples, with a bacterial load $>105$ colony-forming-units $(\mathrm{CFU}) / \mathrm{mL}$ only in 10,2\% of them. Most isolates were positive for Haemophilus influenzae, followed by Streptococcus pneumoniae, Group A $\beta$-hemolytic streptococci and Moraxella catarrhalis. Chest CT scan demonstrated the presence of bronchiectasis in $13 \%$ of the children, of which only one had a positive BAL culture for Haemophilus influenzae. Conclusions. Only in a small subgroup of children, persistent neutrophilic alveolitis was associated with a significant bacterial load and the presence of bronchiectasis. Because most pathogens detected in BAL samples cultures can produce biofilms, caution should be used in inappropriate antibiotic prescription in these patients that, chiefly in those with bronchiectasis, in which chest physiotherapy can be of great benefit.
\end{abstract}

\section{INTRODUCTION}

An uncommon, but potentially serious, cause of respiratory disorders in childhood is represented by the extrinsic tracheal compression due to congenital vascular anomalies within the mediastinum [1]. The most severe forms are usually diagnosed during the neonatal period, while the less severe forms can be detected later in life, when unexplained chronic or recurrent respiratory symptoms, or occasional mild dysphagia, lead to endoscopic evaluations. In two retrospective studies we found that the most prevalent respiratory symptoms associated with this condition were chronic cough, poorly responsive to therapy, and recurrent lower respiratory tract infections (LRTI) $[2,3]$. In the absence of known comorbidities, these symptoms are often misdiagnosed as asthma, non-specific bronchial hyperreactivity and/or gastroesophageal reflux and inappropriate prescription of anti-asthmatic drugs and/or antibiotics often occurs [3,4]. The generation of cough in these children is thought to be induced by mechanical sensory inputs, through rapidly adapting mechanoreceptors $[3,5]$. The recurrence of LRTI in these otherwise healthy children is believed to be related to the dynamic collapse of the airway lumen, associated with malacia, that reduces cough effectiveness and interferes with mucociliary clearance [6-8]. Under-recognition and under-treatment of subclinical protracted bacterial infections may lead to the development of chronic suppurative lung disease and bronchiectasis [8]. With this background a retrospective study was performed to evaluate whether tracheal malacia, secondary to abnormal mediastinal vessels, is associated with persistent lower respiratory tract inflammation and 
infection.

\section{MATERIALS AND METHODS}

\section{Study population}

A retrospective study was performed on records of patients referred to the Gaslini Institute because of recurrent/chronic cough and found to have tracheomalacia associated with mediastinal vascular anomalies.

They all had history of recurrent LRTI $(>3 / y)$ treated with antibiotics but were evaluated while in stable condition, at least four months after the last infection. We excluded children presenting other congenital anomalies or malformations, swallowing disorders, primary ciliary dyskinesia, cystic fibrosis and immunodeficiencies.

\section{Patient evaluation}

Demographic data were collected, and physical examination, routine blood tests were performed in all patients. Children underwent additional investigation and consultations, when clinically indicated by the presence of "specific cough pointers" [9]. Diagnosis of tracheal compression by mediastinal vascular abnormalities was diagnosed in all patients by flexible bronchoscopy and chest computed tomography with radiocontrast agents.

\section{Fiberoptic bronchoscopy and bronchoalveolar lavage (BAL)}

Fiberoptic bronchoscopy was performed in sedation, with the patient spontaneously breathing but ventilation was assisted manually, when needed. The video bronchoscopes (Olympus BF-XP190, BF-3C160 or BFP190) were introduced through a face mask in a nostril, as described elsewhere [3]. The presence and the characteristics of tracheal narrowing, in inspiration and expiration or during cough, was evaluated during bronchoscopy and reassessed playing back the session videotape. BAL was performed by injecting $3 \times 1 \mathrm{ml} / \mathrm{kg}$ body weight aliquots of sterile saline solution, which were aspirated at a negative pressure of

$40 \mathrm{~mm} \mathrm{Hg}$ in siliconized plastic tubes. Samples of BAL fluid was sent to the laboratory for microbiologic and cytologic examination and total and differential cell counts were recorded as described [10]. Bacterial growth was expressed as colony-forming units $(\mathrm{CFU}) / \mathrm{mL}$.

\section{Computed tomography (CT)}

Multidetector CT was performed with a 64-slice CT scanner (Siemens Somatom Sensation 64, Siemens, Erlangen, Germany) [11]. The intravenous contrast agent (Iomeron 300, Bracco, Milan, Italy) was administered, in all patients, with a power injector via a peripheral vein, and when possible, the examination was performed with breath holding at suspended inspiration, but in younger and noncooperative patients, quiet breathing was tolerated. CT images were interpreted without knowledge of the bronchoscopic findings.

\section{Data analysis}

Descriptive statistics were performed and reported in terms of absolute frequencies or percentages for qualitative data and expressed as mean with standard deviation in parenthesis or in terms of medians, with lower and upper quartiles in brackets, for quantitative data. Comparisons between BAL data in tracheomalacia children and reference values were performed using Mann U Whitney. Data were analysed using "Statistica release eight" (StatSoft Corp., Tulsa, OK), and a difference of $P$ value less than 0.5 was considered as statistically significant

\section{RESULTS}

31 children, 15 males and 16 females, mean age at time of referral of $5.64+3.62$ years, were included in the study. In all children fiberoptic bronchoscopy demonstrated the presence of pulsatile extrinsic compression of the trachea middle third, leading to different degree of narrowing that increased in expiration and, more, during coughing (figure 1). Mucus/mucopurulent secretions and various degree of congestion and 
edema of the airway mucosa were revealed. Chest CT scans demonstrated the presence of aberrant innominate artery (AIA) in 21 children, right aortic arch (RAA) in 4 children, double aortic arch (DAA) in 2 child and AIA associated with RAA in 5 children (figure 2). Analysis of BAL cells demonstrated a significant difference in total cell numbers, corrected per milliliter of BAL fluid recovered [92.03 (70.15-481.39) cells $\mu \mathrm{L}^{-1}$ ], compared to control values in our laboratory [132.15 (35.15-290.74) cells $\left.\mu \mathrm{L}^{-1} ; \mathrm{p}<0.01\right]$. A neutrophilic alveolitis was detected, with 21.50 (10.55-66.10) neutrophil proportion, compared to control values of $1.70 \%(0.60-3.50) \quad(\mathrm{p}<0.01)$, but with normal lymphocyte and eosinophil counts (figure 3). Microbiological analysis of BAL fluid demonstrated bacterial growth in $35.5 \%$ of the children, but with a pathogen load $>10^{5}$ colony-forming units $(\mathrm{CFU}) / \mathrm{mL}$ only in $10,2 \%$ of them. In these cultures, $72.7 \%$ of the isolates wereHaemophilus influenzae, 27.3\% Streptococcus pneumoniaeand, 9.0\% each for Group A $\beta$-hemolytic streptococci (GABHS) and Moraxella catarrhalis (figure 4). Coinfection were present in two isolated, one being positive for Haemophilus influenzae plus SBEGA and the other for Haemophilus influenzae plus $S$. pneumoniae. BAL neutrophilia tended to be higher in children with bacterial load $>10^{3} \mathrm{CFU} / \mathrm{mL}$ (figure 5). Finally, chest CT scan demonstrated the presence of bronchiectasis in $13 \%$ of the children, $5.9+2.2$ years old, of which one had a positive BAL culture $\left(>10^{4} \mathrm{CFU} / \mathrm{mL}\right)$ for

Haemophilus influenzae.

\section{DISCUSSION}

In this retrospective study we have shown that a significant inflammatory reaction at airway level is present in children with airway malacia due to mediastinal vascular anomalies and recurrent respiratory symptoms. A significant neutrophilic alveolitis was detectable by bronchoalveolar lavage thought the procedure was performed when children were in well-being conditions, at least four months after the last respiratory infection. Additionally, microbiological analysis of BAL fluid demonstrated bacterial growth in more than one third of them, but with a pathogen load $>10^{5} \mathrm{CFU} / \mathrm{mL}$ only in a small proportion. Haemophilus influenzae was the pathogen most frequently isolated. In a total of 512 bronchoscopies, airway malacia was diagnosed in 160 children (94 males) at a median age of 4.0 years (range, 0 to 17 years). Airway malacia is not a rare condition in the general paediatric population, as reported by Boogaard and co-workers [12]. Out of total of 512 bronchoscopies performed in children with a median age of 4.0 years, 160 cases of airway malacia were detected and classified as primary in 136 cases and secondary in 24 cases [12]. Airway malacia, inducing a dynamic collapse of the airway lumen, reduces cough effectiveness and interferes with mucociliary clearance, favoring airway plugging by bronchial secretions and possibly bronchiectasis formation [13]. Moreover, in a study in which bronchoscopy was performed to exclude other wheezing diseases in children with "severe chronic asthma" (likely including a group with airway malacia), Fayon M and co-workers, showed lower airway neutrophilia and infection $\left(>10^{4} \mathrm{CFU} / \mathrm{mL}\right)$ with Haemophilus influenzae detectable in $39.5 \%$ of BAL cultures [14]. Chronic wet cough, evidence of bronchitis at bronchoscopy and airway neutrophilic inflammation are the hallmark of protracted bacterial bronchitis (PBB) [15]. In the first study defining this disorder, Chang $\mathrm{AB}$ and co-workers reported that trachea-bronchomalacia was found in $33 \%$ of children with chronic cough and BAL cultures in PBB group were positive in $47 \%$ of samples for Haemophilus influenzae, in $35 \%$ for Streptococcus pneumoniae and in $26 \%$ forMoraxella catarrhalis [16]. More than one organism grew in a significant number of $\mathrm{PBB}$ patients and $48 \%$ of the children had a positive clinical response to 14 days of amoxicillin clavulanate $(22.5 \mathrm{mg} / \mathrm{kg} /$ dose, b.i.d.) $[16,17]$. In a follow-up study, Chang AB group evaluated the 2-year outcomes of $161 \mathrm{PBB}$ children [18]. They found that a great proportion of them (43.5\%) had more than three episodes of bacterial bronchitis per year, and $8.1 \%$ were diagnosed with bronchiectasis (vs the $13 \%$ of our patient group) [18]. Haemophilus influenzae infection was demonstrated in BAL fluid of $85 \%$ of children with bronchiectasis and of $49 \%$ of children without bronchiectasis. Recurrent PBB episodes and high Haemophilus influenzae titers in BAL fluid were major risk factors for bronchiectasis at 2 years followup, suggesting a role of this specific infection in bronchiectasis pathogenesis [18]. In our patient group, $72.7 \%$ of the isolates were Haemophilus influenzae positive but only one child with bronchiectasis had a positive culture for Haemophilus influenzae. Through its ability to form biofilms, Haemophilus influenzae is a chief bacterial pathogen associated with chronic respiratory disorders [19] and subinhibitory concentrations of beta-lactam antibiotics may promote its transformation into the biofilm phenotype, favoring antibiotic 
resistance and increased susceptibility to reinfection [20]. In randomised controlled trial in children with chronic wet cough it was reported that many of the children whose symptom was not cured by 2 weeks of amoxicillin clavulanate had underlying tracheo-bronchomalacia and needed a longer treatment duration before their cough disappeared $[15,21]$. Therefore, in view of high frequency of positive isolates for Haemophilus influenzae but also of the general low bacterial load and the significat coesistence of bronchiectasis, caution should be used in inappropriate antibiotic prescription in patients with secondary airway malacia and recurrent respiratory infections. This approach may be chiefly important in children with bronchiectasis, in which chest physiotherapy can be of great benefit [22]. When possible, antibiotics should be chosen on the basis of sputum or cough-swab culture and sensitivity, adherence to treatment should be monitored and the opportunity to switch from twice to thrice daily administration or to increase the dosage should be taken in consideration in specific cases to lengthen the time at higher minimum inhibitory concentration levels [15].

\section{References}

1. McLaren CA, Elliott MJ, Roebuck DJ. Vascular compression of the airway in children. Paediatr Respir Rev 2008;9:85-94.

2. Gardella C, Girosi D, Rossi GA, Silvestri M, Tomà P, Bava G, Sacco O. Tracheal compression by aberrant innominate artery: clinical presentations in infants and children, indications for surgical correction by aortopexy, and short- and long-term outcome. J Pediatr Surg. 2010;45:564-73.

3. Ghezzi M, Silvestri M, Sacco O, Panigada S, Girosi D, Magnano GM, Rossi GA. Mild tracheal compression by aberrant innominate artery and chronic dry cough in children. Pediatr Pulmonol. 2016;51:286-94.

4. Chang AB, Glomb WB. Guidelines for evaluating chronic cough in pediatrics. ACCP evidence-based clinical practice guidelines. Chest 2006;129:260S-283S.

5. Widdicombe JG. Neurophysiology of the cough reflex. Eur Respir J 1995;8:1193-1202.

6. Carden KA, Boiselle PM, Waltz DA, Ernst A. Tracheomalacia and tracheobronchomalacia in children and adults: an in depth review. Chest 2005;127:984e1005

7. Chang AB, Redding GJ, Everard ML. Chronic wet cough: Protracted bronchitis, chronic suppurative lung disease and bronchiectasis. Pediatr Pulmonol. 2008;43:519-31.

8. Verhagen LM, de Groot R. Recurrent, protracted and persistent lower respiratory tract infection: A neglected clinical entity. J Infect. 2015;71:S106-11.

9. Chang AB, Phelan PD, Sawyer SM, Robertson CF. Airway hyperresponsiveness and cough-receptor sensitivity in children with recurrent cough. Am J Respir Crit Care Med 1997;155:1935-1939.

10. Sacco O, Silvestri M, Ghezzi M, Capizzi A, Rossi GA. Airway inflammation and injury in children with prevalent weakly acidic gastroesophageal refluxes. Respir Med. 2018;143:42-47.

11. Sacco O, Santoro F, Ribera E, Magnano GM, Rossi GA. Short-length ligamentum arteriosum as a cause of congenital narrowing of the left main stem bronchus. Pediatr Pulmonol. 2016;51:1356-1361.

12. Boogaard R, Huijsmans SH, Pijnenburg MW, et al. Tracheomalacia and bronchomalacia in children: incidence and patient characteristics. Chest 2005;128:3391-3397.

13. Santiago-Burruchaga M, Zalacain-Jorge R, Vazquez-Cordero C. Are airways structural abnormalities more frequent in children with recurrent lower respiratory tract infections? Respir Med. 2014;108:800-5.

14. Fayon M, Just J, Thien HV, et al. Bacterial flora of the lower respiratory tract in children with bronchial asthma. Acta Paediatr 1999;88:1216-1222.

15. Chang AB, Upham JW, Masters IB, Redding GR, Gibson PG, Marchant JM, Grimwood K. Protracted bacterial bronchitis: The last decade and the road ahead. Pediatr Pulmonol. 2016;51:225-42. 
16. Marchant JM, Masters IB, Taylor SM, Cox NC, Seymour GJ, Chang AB. Evaluation and outcome of young children with chronic cough. Chest. 2006;129:1132-41.

17. Wurzel DF, Marchant JM, Yerkovich ST, Upham JW, Mackay IM, Masters IB, Chang AB. Prospective characterization of protracted bacterial bronchitis in children. Chest. 2014;145:1271-1278.

18. Wurzel DF, Marchant JM, Yerkovich ST, et al. Protracted bacterial bronchitis in children: natural history and risk factors for bronchiectasis. Chest. 2016;150:1101-1108.

19. Sacco O, Capizzi AF, Silvestri M, Rossi GA. Recurrence of Protracted Bacterial Bronchitis in Children: What Can We Do? Chest. 2017;151:940.

20. Wu S, Li X, Gunawardana M, Maguire K, et al. Beta-lactam antibiotics stimulate biofilm formation in non-typeable Haemophilus influenzae by up-regulating carbohydrate metabolism. PLoS One. 2014;9:e99204.

21. Marchant J, Masters IB, Champion A, Petsky H, Chang AB. Randomised controlled trial of amoxycillin clavulanate in children with chronic wet cough. Thorax. 2012;67:689-93.

22. Chang AB, Marchant JM. Protracted bacterial bronchitis is a precursor for bronchiectasis in children: myth or maxim? Breathe (Sheff). 2019;15:167-170.

\section{Figure legends}

Figure 1. CT scan and bronchoscopic images of a 9 years old child with extrinsic tracheomalacia due to compression by aberrant innominate artery. A. CT scans show the anomalous course of the innominate artery (white arrows) that causes an extrinisic compression on the tracheal wall inducing the

reduction of the tracheal caliber (asterisk). B. Bronchoscopic image in quiet breth showing the significant reduction tracheal caliber due both to the extrinisic compression and the bulging of the posterior membranous wall. C. Bronchoscopic image during coughing, showing collapse of the tracheal walls and the almost complete obstruction of the lumen.

Figure 2. Distribution of children with secondary tracheomalacia induced different mediastinal vessel abnormalities. (A) Number of patients with aberrant innominate artery (AIA), right aortic arch (RAA), double aortic arch (DAA), and AIA and RAA in the whole population.

Figure 3. Bronchoalveolar lavage (BAL) cell data in children with secondary tracheomalacia induced mediastinal vessel abnormalities (TM children), white bars, and normal reference value (gray bars). Data are presented as median values, interquartile range and min-max.

Figure 4. Percentage of bacteria grown in bronchoalveolar lavage sample cultures data in children with secondary tracheomalacia induced mediastinal vessel abnormalities: Haemophilus influenzae ,Streptococcus pneumoniae, Group A $\beta$-hemolytic streptococci (GABHS) and Moraxella catarrhalis .

Figure 5. Neutrophilic inflammation and bacterial load in bronchoalveolar lavage sample cultures. The percentage of BAL neutrophils is expressed on the ordinate and the colony-forming units $(\mathrm{CFU}) / \mathrm{mL}$ on the abscissa.
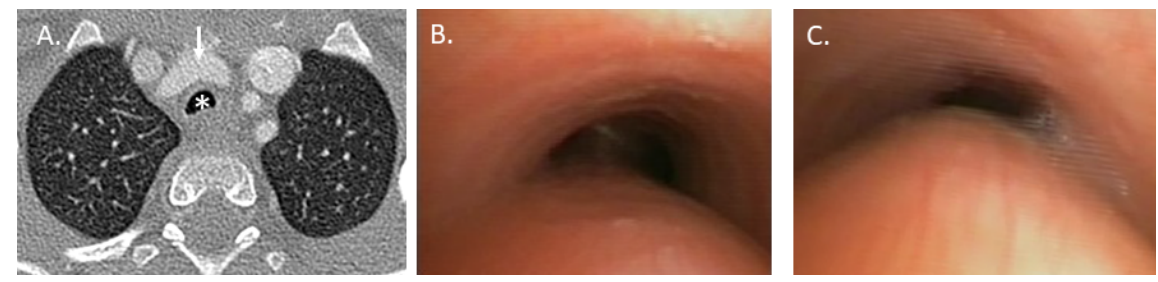

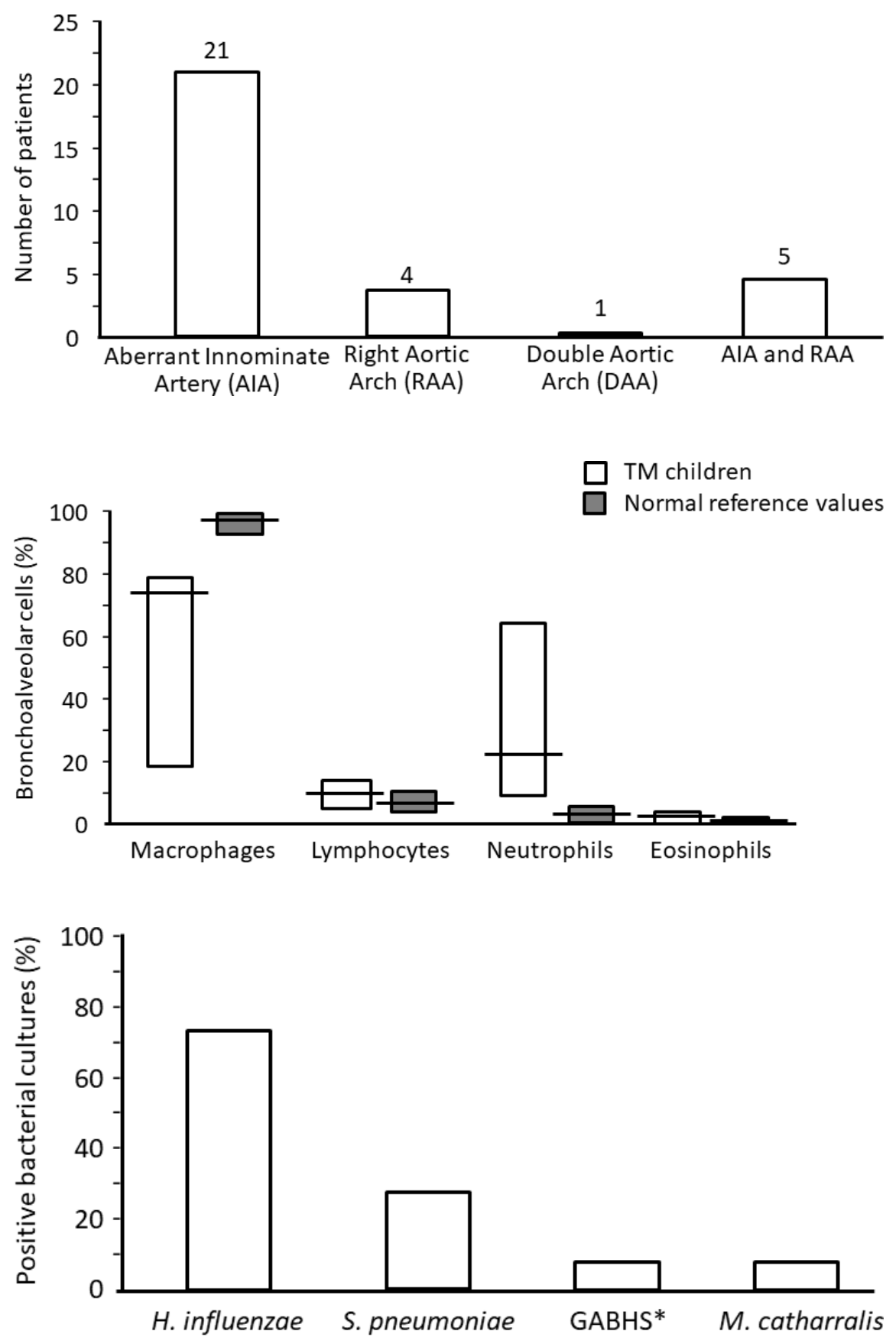

* GABHS: group A $\beta$-hemolytic streptococci 


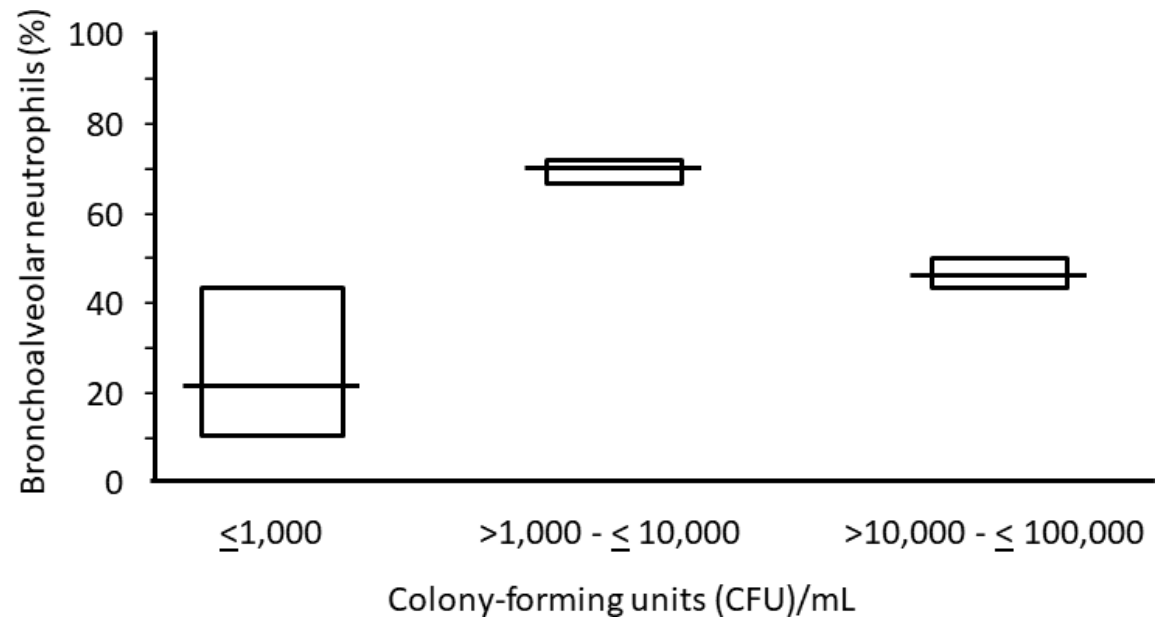

